\title{
Patient-Reported Care Coordination: Associations With Primary Care Continuity and Specialty Care Use
}

\author{
David T. Liss, $M A^{1,2}$ \\ Jessica Cbubak, $\mathrm{PbD}^{2,3}$ \\ Melissa L. Anderson, MS \\ Katbleen W. Saunders, JD2 \\ Leab Tuzzio, $\mathrm{MPH}^{2}$ \\ Robert J. Reid, $M D P b D^{2}$
}

'Department of Health Services, University of Washington, Seattle, Washington

${ }^{2}$ Group Health Research Institute, Seattle, Washington

${ }^{3}$ Department of Epidemiology, University of Washington, Seattle, Washington

\begin{abstract}
PURPOSE Care coordination is increasingly recognized as a necessary element of high-quality, patient-centered care. This study investigated (1) the association between care coordination and continuity of primary care, and (2) differences in this association by level of specialty care use.
\end{abstract}

METHODS We conducted a cross-sectional study of Medicare enrollees with select chronic conditions in an integrated health care delivery system in Washington State. We collected survey information on patient experiences and automated health care utilization data for 1 year preceding survey completion. Coordination was defined by the coordination measure from the short form of the Ambulatory Care Experiences Survey (ACES). Continuity was measured by primary care visit concentration. Patients who had 10 or more specialty care visits were classified as high users. Linear regression was used to estimate the association between coordination and continuity, controlling for potential confounders and clustering within clinicians. We used a continuity-by-specialty interaction term to determine whether the continuity-coordination association was modified by high specialty care use.

RESULTS Among low specialty care users, an increase of 1 standard deviation (SD) in continuity was associated with an increase of 2.71 in the ACES coordination scale $(P<.001)$. In high specialty care users, we observed no association between continuity and reported coordination $(P=.77)$.

CONCLUSIONS High use of specialty care may strain the ability of primary care clinicians to coordinate care effectively. Future studies should investigate care coordination interventions that allow for appropriate specialty care referrals without diminishing the ability of primary care physicians to manage overall patient care.

Ann Fam Med 2011;9:323-329. doi:10.1370/afm.1278.

\section{INTRODUCTION}

$\mathrm{D}$ elivering the full array of evidence-based primary care to typical practice populations is an increasingly complex and time-consuming task, ${ }^{1-4}$ particularly for patients with one or more chronic conditions. ${ }^{5,6}$ Primary care clinicians may have neither sufficient time nor sufficient expertise to meet all of their patients' clinical needs, often necessitating consultation with specialist physicians and referral to communitybased programs and other types of service organizations. ${ }^{2,7}$

As care is increasingly comingled between primary care physicians and specialists, ${ }^{8}$ patients, payers, and professional organizations have recognized the need for better strategies to coordinate care across settings and physicians. ${ }^{9-12}$ Though no single model of care coordination is universally applicable across patient populations ${ }_{1}^{13}$ previous research has found clinical and economic benefits associated with numerous care coordination interventions. ${ }^{10,14-17}$ 
In primary care, continuity of care has been defined as the bridging relationship between a single practitioner and a patient that extends beyond specific episodes of illness or disease. ${ }^{18-21}$ By facilitating clinical information transfer and coherent management of patient needs over time, ${ }^{22}$ continuity may be a driver of care coordination across primary care visits and across care settings. Our limited understanding of the association between continuity and coordination ${ }^{13,22-24}$ provided motivation for the current study.

This cross-sectional study was conducted in an elderly patient population with select chronic conditions.

It investigated the association between primary care continuity - defined as the concentration of visits to clinicians in the primary care setting - and patients' reports of coordination processes by primary care clinicians. This project also investigated the links between the association under study and patients' use of outpatient specialty care. We hypothesized that primary care continuity would be positively associated with patient-reported coordination of care conducted by primary care clinicians. We also hypothesized that high outpatient use of medical specialists may increase the coordination demands for primary care clinicians, thereby attenuating the association under study among high specialty users

\section{METHODS}

\section{Data Collection}

This project was a secondary data analysis of crosssectional data collected as part of a larger research study on the care experience provided to Medicare enrollees with select chronic conditions at Group Health, an integrated health care delivery system in Washington State and Idaho. During March through September 2008, data were collected from questionnaires mailed to Group Health members who were eligible to enroll in a new Medicare Advantage Special Needs Plan offered to elderly patients with chronic conditions. ${ }^{25}$ Eligible patients were aged 65 years or older, received care at a Group Health clinic in King County or Pierce County in western Washington State, and had at least 1 of the following chronic conditions: diabetes, coronary artery disease (CAD), or congestive heart failure (CHF). The questionnaires elicited data on patient demographics, self-rated health, care experiences with individual physicians, ${ }^{26}$ care for chronic conditions, ${ }^{27}$ and patient activation. ${ }^{28,29}$
A chronic disease was considered to be present if the patient received a diagnosis code for a qualifying study condition during the 24 months preceding survey data collection. The Group Health Institutional Review Board approved all study protocols.

Automated health care utilization data were extracted for the 1 -year period preceding data collection. The automated data capture diagnoses, procedures, and other data for all ambulatory and inpatient episodes of care provided at Group Health or through contract arrangements. Data from Group Health's automated databases have been extensively validated and used in studies of health care utilization. ${ }^{30-35} \mathrm{We}$ extracted data on patients' inpatient and outpatient visits, as well as patient age, sex, paneled primary care physician at the date of questionnaire return, and RxRisk comorbidity score, an automated risk adjustment measure computed from outpatient prescription data in which higher RxRisk scores indicating higher expected health care cost. ${ }^{36}$

\section{Coordination of Care}

The dependent variable in this study is the coordination of care summary measure from the short form version of the Ambulatory Care Experiences Survey (ACES) ${ }^{26}$ Items in this subscale address the care received at a personal doctor's office, both from the personal physician and from other physicians or nurses at this office. A screening item first asked, "In the last 12 months, are there other doctors or nurses in your personal doctor's office who you have seen for any of your visits?" A follow-up item was asked of respondents who answered yes or did not answer the screening item, and 2 additional items were asked of all respondents (Figure 1). Using established survey scoring guidelines, ${ }^{37}$ we then calculated the coordination measure by rescaling respondents' raw item scores-using responses from 2 
or 3 items, depending on the response-into a continuous 100-point scale in which higher scores indicated higher coordination. Coordination scores were considered missing for respondents who did not answer more than 1 indicated item on the questionnaire.

\section{Continuity of Care}

We constructed our primary independent variable, the continuous Bice-Boxerman measure of continuity of care ${ }^{38}$ using outpatient primary care visit data from the 1 -year period preceding each respondent's date of questionnaire return. This measure of the concentration of care across primary care clinicians ranges from 0 to 1 , with increasing scores indicating higher continuity. The measure incorporates the number of primary care clinicians seen during a defined follow-up period, as well as the absolute number of in-person visits with each clinician. It was calculated as follows:

$$
\text { Continuity }=\frac{\sum_{j=1}^{s} n_{j}{ }^{2}-N}{N(N-1)} .
$$

where $N$ is the total number of primary care visits, $n_{j}$ is the number of visits to clinician $j$ during the study period, and $s$ is the number of primary care clinicians seen by the patient. Qualifying primary care visits included visits to the following clinicians that occurred in primary care settings: family practitioners, general internists, advanced registered nurse practitioners, and physician assistants.

The Bice-Boxerman measure is either uninformative or unstable for patients with few clinician visits. Among patients with many visits, however, this measure has been shown to be unrelated to utilization levels. ${ }^{39} \mathrm{We}$ therefore chose to limit our analyses to study respondents who had 3 or more primary care visits during the study period.

\section{Analysis}

We used $\chi^{2}$ and Student's $t$ tests to compare demographic and health characteristics of respondents and nonrespondents. In the analytic sample, we used 1 -way analysis of variance (ANOVA) to test for differences in mean care coordination scores between covariate groups. We conducted a multivariate linear regression analysis to assess the association between care coordination (outcome) and the Bice-Boxerman continuity measure (exposure). The continuity measure was included in regression models as the raw score divided by the standard deviation (SD), leading to a model coefficient interpreted as the difference in mean coordination score associated with an increase of 1 standard deviation in continuity.

To determine whether the association between care coordination and continuity was modified by level of specialty use, an indicator for high specialty care use (10 or more specialist visits during the 1-year study period) and an interaction between high specialty care use and care continuity were included in the model. Because no reference standard exists to identify high users of care, ${ }^{40}$ we based our definition of high use on examples from the literature ${ }^{40,41}$ and conducted sensitivity analyses across a range of thresholds.

Other covariates included in the model to adjust for potential confounding were age (65-69, 70-74, 75-79, 80-84, 85+ years), sex, race (white, nonwhite), chronic disease status (CAD only, diabetes only, both CAD and diabetes), self-rated health (excellent/very good, good, fair/poor), an indicator of whether the respondent had any hospital admissions-which may reflect increased illness burden and an increased need for care coordination - and tertile of RxRisk score (low, medium, high) based on the empirical RxRisk distribution in the final analytic sample.

Generalized linear models with identity link, Gaussian error distribution, and independent correlation structures were fit using generalized estimating equations to control for clustering of respondents within their paneled primary care physician. Robust sandwich variance estimates were used to ensure asymptotically correct variance estimates in the case of model misspecification. ${ }^{42}$ Analyses were conducted in Stata 11.0 (StataCorp, LP, College Station, Texas).

\section{RESULTS}

The patient questionnaire was sent to 4,658 elderly patients with qualifying chronic conditions, 3,224 (69\%) of whom returned the questionnaires. Fortyseven were ineligible because a proxy individual had completed the questionnaire, they had not been enrolled for a full year prior to returning the questionnaire, or they did not meet study requirements for age or chronic disease. Two-hundred eighteen of those who completed the questionnaire $(7 \%)$ declined authorization to access medical record data for final analyses. Compared with the 2,959 respondents who completed the questionnaire and consented to medical record linkage, nonparticipants (nonresponders and ineligible respondents, $\mathrm{n}=1,699)$ were older $(P<.001)$ and more often female $(P<.001)$.

The analysis was limited to respondents with 3 or more primary care visits in the study year to ensure a meaningful measure of care continuity. ${ }^{39}$ Compared with those with 0 to 2 primary care visits $(n=806)$, respondents with 3 or more visits $(n=2,153,73 \%)$ were more often female, more likely to have both CAD and diabetes, older, had worse self-reported health, higher RxRisk scores, and more likely to have been hospital- 
ized during the past year $(P<.01$; data not shown).

Reported care coordination appeared higher for those with 0 to 2 visits than those with 3 or more visits (mean 79.1 vs 77.7), but the difference was not statistically significant $(P=.13)$.

After identifying respondents who met all inclusion criteria, we excluded an additional 102 respondents with missing data on the dependent coordination measure. All 795 respondents with a CHF diagnosis had coexisting CAD, and inclusion of a CHF indicator term in regression models did not affect study findings (data not shown), leading us to omit CHF as a category in the chronic disease status covariate.

The final analytic sample $(n=2,051)$ had a mean coordination score of $77.7(\mathrm{SD}=20.9)$ and a mean Bice-Boxerman continuity of care index of 0.55 $(\mathrm{SD}=0.32)$. The final sample was majority female, predominantly white, and had a mean age of 80 years (Table 1). Roughly one-fifth of respondents had both CAD and diabetes, and $37 \%$ rated their health as fair or poor. One-way ANOVA tests found differences in mean coordination scores by sex, race, and self-rated health $(P<.01)$. Compared with low specialty care users, high users were more often male, white, and had CAD $(P<.01)$ High specialty care users also had conditions that were more clinically complex, as evidenced by lower self-rated health, higher RxRisk scores, and higher rates of hospital admission in the past year $(P<.01)$.

We used multivariate linear regression models to assess the relationship between continuity and coordination. To determine whether specialty care use was an independent predictor of care coordination, we initially included high specialty care use as a covariate in regression models containing no interaction terms (Table 2). In an adjusted model controlling for demographics and patient health, an increase of $1 \mathrm{SD}$ in continuity was associated with an increase of 2.21 in the coordination measure $(P$ $<.001$, Table 2), while no association was observed between high specialty care use and coordination $(P=.27)$. White race $(P=.004)$, increased self-rated health $(P<.001)$, and male sex $(P=.001)$ were positively associated with coordination.

We examined whether specialty care use modified the association between continuity and coordination by including a continuity-by-specialty interaction in the final statistical model. The interaction term suggested differences in reported coordination between specialty care use groups (coefficient $=-2.42, P=.03$ ), leading us to report results separately for low and high specialty care users. Among low specialty care users, an increase of 1 standard deviation in continuity of care was associated with an increase of 2.71 in coordination $(P<.001$, Table 3$)$. Among respondents with high specialty care use, however, the model showed no association between continuity and reported coordination $(P=.77)$. As we observed in the previous multi-

\begin{tabular}{|c|c|c|c|c|}
\hline \multirow[b]{2}{*}{ Characteristics } & \multicolumn{2}{|c|}{$\begin{array}{l}\text { Specialty Care Use } \\
\text { (No. of Visits) }{ }^{a}\end{array}$} & \multirow[b]{2}{*}{$\begin{array}{l}\text { Total, } \\
\text { N (\%) }\end{array}$} & \multirow{2}{*}{$\begin{array}{c}\text { Coordination } \\
\text { Score } \\
\text { Mean (SD) }\end{array}$} \\
\hline & $\begin{array}{l}\text { Low }(0-9) \\
\text { n (\%) }\end{array}$ & $\begin{array}{l}\text { High (10+) } \\
\text { n (\%) }\end{array}$ & & \\
\hline Total & $1,552(76)$ & $499(24)$ & $2,051(100)$ & $77.7(21)$ \\
\hline Sex & & c & & d \\
\hline Female & $910(59)$ & $259(52)$ & $1,169(57)$ & $76.2(22)$ \\
\hline Male & $642(41)$ & $240(48)$ & $882(43)$ & $79.6(19)$ \\
\hline Race & & d & & c \\
\hline White & $1,312(85)$ & $461(93)$ & $1,773(87)$ & $78.2(20)$ \\
\hline Nonwhite & $231(15)$ & $35(7)$ & $266(13)$ & $74.1(23)$ \\
\hline \multicolumn{5}{|l|}{ Age, y } \\
\hline $65-69$ & $158(10)$ & $43(8)$ & $201(10)$ & $75.3(22)$ \\
\hline $70-74$ & $243(16)$ & $68(14)$ & $311(15)$ & $77.7(20)$ \\
\hline $75-79$ & $330(21)$ & $121(24)$ & $451(22)$ & $79.5(20)$ \\
\hline $80-84$ & $379(24)$ & $128(26)$ & $507(25)$ & $77.0(21)$ \\
\hline $85+$ & $442(29)$ & $139(28)$ & $581(28)$ & $77.7(21)$ \\
\hline Chronic disease & & d & & \\
\hline CAD only & $564(36)$ & $231(46)$ & 795 (39) & $77.2(21)$ \\
\hline Diabetes only & $697(45)$ & $132(27)$ & $829(40)$ & $78.5(21)$ \\
\hline Both CAD and diabetes & 291 (19) & $136(27)$ & $427(21)$ & $77.1(21)$ \\
\hline Self-rated health & & d & & d \\
\hline Excellent/very good & $321(21)$ & $75(16)$ & $396(20)$ & $82.6(19)$ \\
\hline Good & $669(44)$ & $195(40)$ & $864(43)$ & $78.2(20)$ \\
\hline Fair/poor & $519(35)$ & $213(44)$ & $732(37)$ & $74.4(22)$ \\
\hline RxRisk & & d & & \\
\hline Low & $539(35)$ & $125(25)$ & $664(33)$ & $77.4(21)$ \\
\hline Medium & $549(35)$ & $148(30)$ & $697(34)$ & $77.9(21)$ \\
\hline High & $460(30)$ & $222(45)$ & $682(33)$ & $77.8(20)$ \\
\hline Any hospitalizations & & d & & \\
\hline Yes & $305(20)$ & $252(51)$ & $557(27)$ & $76.4(21)$ \\
\hline No & $1,247(80)$ & $247(49)$ & $1,494(73)$ & $78.2(21)$ \\
\hline \multicolumn{5}{|c|}{$C A D=$ coronary artery disease $;$ RxRisk = medication-based risk adjustment measure.$^{36}$} \\
\hline \multicolumn{5}{|c|}{$\begin{array}{l}\text { a } \chi^{2} \text { Test compared percentages between low and high specialty care use groups. } \\
\text { b One-way analysis of variance compared mean coordination across covariate groups. } \\
\text { c } P<.01 \text {. } \\
\text { d } P<.001 \text {. }\end{array}$} \\
\hline
\end{tabular}


variate model, white race, increased self-rated health, and male sex were again positively associated with coordination at $P<.05$ (data not shown).

We conducted sensitivity analyses to assess whether results were robust to changes in our empirical definition of high specialty care use. To incorporate a range of existing definitions of high users of care, ${ }^{40}$

\begin{tabular}{|c|c|c|}
\hline Variables & $\begin{array}{l}\text { Difference in } \\
\text { Mean Coordination } \\
(95 \% \mathrm{Cl})\end{array}$ & $P$ Value \\
\hline \multicolumn{3}{|l|}{ Independent, unadjusted } \\
\hline Continuity, raw score/SD & $2.22(1.3$ to 3.1$)$ & $<.001$ \\
\hline High specialty care use & $0.57(-1.5$ to 2.6$)$ & .59 \\
\hline \multicolumn{3}{|l|}{ Independent, adjusted ${ }^{\mathrm{a}}$} \\
\hline Continuity, raw score/SDb & $2.21(1.2$ to 3.2$)$ & $<.001$ \\
\hline High specialty care use & $1.23(-1.0$ to 3.4$)$ & .27 \\
\hline \multicolumn{3}{|l|}{ Covariates } \\
\hline Female & $-2.71(-4.3$ to -1.1$)$ & .001 \\
\hline White & 3.97 (1.3 to 6.7$)$ & .004 \\
\hline Age (referent: 65-69), y & & .03 \\
\hline $70-74$ & $3.63(-0.1$ to 7.4$)$ & \\
\hline $75-79$ & $5.46(1.6$ to 9.3$)$ & \\
\hline $80-84$ & $2.89(-1.0$ to 6.8$)$ & \\
\hline $85+$ & $4.67(1.0$ to 8.3$)$ & \\
\hline Chronic disease (referent: CAD) & & .27 \\
\hline Diabetes & $1.96(-0.4$ to 4.4$)$ & \\
\hline Both CAD and diabetes & $1.08(-1.7$ to 3.8$)$ & \\
\hline Self-rated health (referent: good) & & $<.001$ \\
\hline Excellent/very good & 4.55 (2.1 to 7.0$)$ & \\
\hline Fair/poor & $-3.42(-5.4$ to -1.5$)$ & \\
\hline RxRisk score (referent: medium) & & .53 \\
\hline Low & $-1.21(-3.5$ to 1.1$)$ & \\
\hline High & $0.03(-2.3$ to 2.4$)$ & \\
\hline Any hospitalizations & $-1.64(-3.6$ to 0.4$)$ & .11 \\
\hline \multicolumn{3}{|c|}{$\mathrm{CAD}=$ coronary artery disease; RxRisk = medication-based risk adjustment measure. ${ }^{36}$} \\
\hline \multicolumn{3}{|c|}{$\begin{array}{l}\text { a Excluding } 77 \text { respondents because of missing race, self-rated health, or primary care } \\
\text { physician data. } \\
\text { b Interpretation: mean predicted change in coordination associated with increase of } 1 \mathrm{SD} \\
\text { (mean continuity }=0.55 ; \mathrm{SD}=0.32 \text { ). }\end{array}$} \\
\hline
\end{tabular}

Table 3. Association Between Coordination and Continuity According to Level of Specialty Care Use

\begin{tabular}{|c|c|c|}
\hline Independent Variables (Adjusted) ${ }^{a, b}$ & $\begin{array}{c}\text { Difference in } \\
\text { Mean Coordination } \\
(95 \% \mathrm{Cl})\end{array}$ & $\begin{array}{c}P \\
\text { Value }\end{array}$ \\
\hline Continuity in low specialty care users, raw score $(S D)^{c}$ & $2.71(1.6$ to 3.8$)$ & $<.001$ \\
\hline Continuity in high specialty care users, raw score (SD) ${ }^{c}$ & $0.28(-1.6$ to 2.2$)$ & .77 \\
\hline \multicolumn{3}{|c|}{$\mathrm{CAD}=$ coronary artery disease $;$ RxRisk $=$ medication-based risk adjustment measure..$^{36}$} \\
\hline \multicolumn{3}{|c|}{$\begin{array}{l}\text { adjusted for sex, race (nonwhite, white), age (65-69, 70-74, 75-79, 80-84, } 85+\text { years), chronic disease } \\
\text { (CAD, diabetes, both), self-rated health (excellent/very good, good, fair/poor), RxRisk (low, medium, high), } \\
\text { hospital admissions (no, yes). } \\
{ }^{b} \text { Excluded } 77 \text { respondents because of missing race, self-rated health, or primary care physician data. } \\
\text { c Interpretation: mean predicted change in coordination associated with increase of } 1 \text { SD. }\end{array}$} \\
\hline
\end{tabular}

we varied the threshold of high utilization from 8 to 12 annual specialty care visits. Findings were stable across this range of high specialty care use; the association under study remained positive and statistically significant for patients with low specialty care use, with no association observed in high specialty care users.

\section{DISCUSSION}

We observed a positive association between primary care continuity and patient-reported care coordination among study respondents with 3 or more primary care visits and low levels of outpatient specialty care use, but not among respondents defined as high users of specialty care. Sensitivity analyses were robust across a range of high specialty care use thresholds.

Results are informative with respect to the links between care coordination and continuity of care in the elderly population under study. Study findings seem to shed light on a natural tension between the provision of specialty care and primary care clinicians' ability to plan and coordinate patient care activities. As patients receive more specialty care, a high number of visits to medical specialists may strain the ability of primary care clinicians to effectively coordinate care. Although high specialty care use may be a proxy for clinical complexity-which inherently presents challenges to effective primary care delivery - visits to specialists add further complexity to care delivery processes, creating additional opportunities for gaps in coordination to occur.

Prior research has identified 3 types of care continuity-in relationships, information, and management ${ }^{21,22}$ — none of which are explicitly measured by the Bice-Boxerman measure used in this study. These 3 forms of continuity may assist in interpreting apparent coordination

gaps observed among respondents with high specialty care use. When patients increase their specialty care use, primary care clinicians presumably play a diminished role in directing overall treatment plans, leading to perceived lapses in coordination. Although electronic information systems provided the means to transfer information between primary and specialty care clinicians in this study setting, they were not able to completely mitigate these gaps. As the locus of care management expands 
outside the primary care setting to include medical specialists, it becomes more difficult for primary care clinicians to consistently direct a care plan in which patients comfortably and regularly interact with primary care clinicians.

There are several limitations to this study. Group Health's integrated delivery structure-in which patients are paneled with individual primary care physicians and receive much of their care at clinics owned and operated by Group Health-currently differs from that of most American health care settings. Whereas the integration of Group Health clinical databases ensures continuity of information transfer, primary care clinicians in other settings may face additional barriers to care coordination via decreased access to specialty care visit data. This study was conducted in a high-need elderly population with 3 or more annual primary care visits, and findings may not be applicable to patient populations with different medical needs, utilization patterns, or reduced access to comprehensive primary care services.

There are also inherent limitations to the crosssectional study design and the variables under study. As in all observational studies, we were able to estimate associations and not necessarily causal effects. Differences between high and low users of specialty care, such as the increased morbidity burden of high specialty care users, may have affected findings in ways we were unable to observe or control. Although patients' generally high coordination reports are consistent with previous findings, ${ }^{43}$ it is unclear whether statistically significant effect sizes reflect clinically meaningful changes in coordination. The exploratory nature of this analysis requires both caution in the interpretation of findings and validation in other patient populations and clinical settings.

If validated elsewhere, our findings suggest that visit-based continuity within the primary care setting is an insufficient facilitator of coordination for clinically complex patients with high specialty care use. Future research efforts should investigate processes that foster continuity in management and relationships. For example, active collaboration between primary care clinicians and specialists may be fostered by interoffice initiatives, such as formal service agreements, ${ }^{44,45}$ electronic referrals, ${ }^{46,47}$ and remote or in-person specialist consultations. ${ }^{48,49}$ Coordination for these patients may also be facilitated by chronic care quality reports, adequate visit time with patients, and nurse support for chronic care monitoring, which were associated with communication on referrals in a recent, nationally representative survey of primary care physicians and specialists. ${ }^{50}$

When elderly patients with chronic conditions receive large amounts of outpatient specialty care, the ability of primary care clinicians to coordinate care in the traditional office setting seems to diminish. As patients continue to live with increasing amounts of chronic disease, ${ }^{51}$ this challenge to effective care coordination will only grow more acute, requiring new methods of care provision to preempt gaps in continuity and coordination.

To read or post commentaries in response to this article, see it online at http://www.annfammed.org/cgi/content/full/9/4/323.

Key words: Care coordination; continuity of patient care; ambulatory care; primary health care

Submitted December 14, 2010; submitted, revised, March 14, 2011; accepted April 11, 2011.

Funding support: This project was supported by Group Health Research Institute Development Funds (Chubak). David Liss received support under traineeships from the Agency for Healthcare Research and Quality (AHRQ \#T32 HS013853) and the National Center for Research Resources (NCRR \#TL1 RR025016).

Versions of this study this study were presented th the AHRQ NRSA Trainees Research Conference, June 26, 2010, Boston, Massachusetts; and at the 17th Annual AHRQ NRSA Trainees Research Conference, June 11, 2011, Seattle, Washington.

Acknowledgments: We wish to thank Charles Maynard, PhD, and Allen Cheadle, PhD, for their assistance during analysis planning and drafting of the manuscript.

\section{References}

1. Yarnall KS, Pollak KI, Østbye T, Krause KM, Michener JL. Primary care: is there enough time for prevention? Am J Public Health. 2003;93(4):635-641.

2. Østbye T, Yarnall KS, Krause KM, Pollak KI, Gradison M, Michener $\mathrm{JL}$. Is there time for management of patients with chronic diseases in primary care? Ann Fam Med. 2005;3(3):209-214.

3. Mechanic D, McAlpine DD, Rosenthal M. Are patients' office visits with physicians getting shorter? N Engl J Med. 2001;344(3):198-204.

4. Campion EW. A symptom of discontent. N Engl J Med. 2001;344(3): 223-225.

5. Grumbach K, Bodenheimer T. A primary care home for Americans: putting the house in order. JAMA. 2002;288(7):889-893.

6. Wagner EH, Austin BT, Von Korff M. Organizing care for patients with chronic illness. Milbank Q. 1996;74(4):511-544.

7. Bodenheimer T, Lo B, Casalino L. Primary care physicians should be coordinators, not gatekeepers. JAMA. 1999;281(21):2045-2049.

8. Schappert SM, Rechtsteiner EA. Ambulatory medical care utilization estimates for 2006. Natl Health Stat Report. Aug 6 2008(8):1-29.

9. The Commonwealth Fund Survey of Public Views of the US Health Care System. 2006; http://www.cmwf.org/surveys/surveys_show. htm?doc_id=394593. Accessed Sep 24, 2010.

10. Peikes D, Chen A, Schore J, Brown R. Effects of care coordination on hospitalization, quality of care, and health care expenditures among Medicare beneficiaries: 15 randomized trials. JAMA. 2009; 301(6):603-618

11. Institute of Medicine. Crossing the Quality Chasm. A New Health System for the Twenty-First Century. Washington, DC: Institute of Medicine;2001. 
12. American Academy of Family Physicians, American Academy of Pediatrics, American College of Physicians, American Osteopathic Association. Joint Principles of the Patient-Centered Medical Home. 2007; http:// www.pcpcc.net/content/joint-principles-patient-centered-medicalhome. Accessed Jan 10, 2010.

13. McDonald KM, Sundaram V, Bravata DM, et al. Care Coordination. Technical Review 9. In: Shojania KG MK, Wachter RM, Owens DK, eds. Closing the Quality Gap: A Critical Analysis of Quality Improvement Strategies. Vol 7. Rockville, MD: Agency for Healthcare Research and Quality (AHRQ); 2007.

14. Shojania KG, Ranji SR, McDonald KM, et al. Effects of quality improvement strategies for type 2 diabetes on glycemic control: a meta-regression analysis. JAMA. 2006;296(4):427-440

15. Langhorne P, Taylor G, Murray G, et al. Early supported discharge services for stroke patients: a meta-analysis of individual patients' data. Lancet. 2005;365(9458):501-506.

16. Gilbody S, Whitty P, Grimshaw J, Thomas R. Educational and organizational interventions to improve the management of depression in primary care: a systematic review. JAMA. 2003;289(23):3145-3151.

17. Windham BG, Bennett RG, Gottlieb S. Care management interventions for older patients with congestive heart failure. Am J Manag Care. 2003;9(6):447-459; quiz 460-441.

18. Hennen BK. Continuity of care in family practice. Part 1: dimensions of continuity. J Fam Pract. 1975;2(5):371-372.

19. Rogers J, Curtis P. The concept and measurement of continuity in primary care. Am J Public Health. 1980;70(2):122-127.

20. Hjortdahl P. Ideology and reality of continuity of care. Fam Med. 1990;22(5):361-364

21. Haggerty JL, Reid RJ, Freeman GK, Starfield BH, Adair CE, McKendry R. Continuity of care: a multidisciplinary review. BMJ. 2003; 327(7425):1219-1221.

22. Reid R, McKendry R, Haggerty J. Defusing the Confusion: Concepts and Measures of Continuity of Health Care: Final Report. Ottawa, ON: Canadian Health Services Research Foundation; 2002.

23. Ware NC, Tugenberg T, Dickey B. Ethnography and measurement in mental health: qualitative validation of a measure of continuity of care (CONNECT). Qual Health Res. 2003;13(10):1393-1406.

24. Christakis DA. Does continuity of care matter? Yes: consistent contact with a physician improves outcomes. West J Med. 2001;175(1):4

25. Centers for Medicare and Medicaid Services. Special Needs Plans. Overview. https://www.cms.gov/SpecialNeedsPlans/. Accessed Aug 28, 2010.

26. Safran DG, Karp M, Coltin K, et al. Measuring patients' experiences with individual primary care physicians. Results of a statewide demonstration project. J Gen Intern Med. 2006;21(1):13-21.

27. Glasgow RE, Wagner EH, Schaefer J, Mahoney LD, Reid RJ, Greene SM. Development and validation of the Patient Assessment of Chronic Illness Care (PACIC). Med Care. 2005;43(5):436-444.

28. Hibbard JH, Stockard J, Mahoney ER, Tusler M. Development of the Patient Activation Measure (PAM): conceptualizing and measuring activation in patients and consumers. Health Serv Res. 2004;39(4 Pt 1):1005-1026

29. Hibbard JH, Mahoney ER, Stockard J, Tusler M. Development and testing of a short form of the patient activation measure. Health Serv Res. 2005;40(6 Pt 1):1918-1930.

30. Fishman PA, Wagner EH. Managed care data and public health: the experience of Group Health Cooperative of Puget Sound. Annu Rev Public Health. 1998;19:477-491.

31. Boudreau DM, Doescher MP, Saver BG, Jackson JE, Fishman PA. Reliability of Group Health Cooperative automated pharmacy data by drug benefit status. Pharmacoepidemiol Drug Saf. 2005;14(12): 877-884.
32. Lozano P, Fishman P, VonKorff M, Hecht J. Health care utilization and cost among children with asthma who were enrolled in a health maintenance organization. Pediatrics. 1997;99(6):757-764.

33. Wagner EH, Sandhu N, Newton KM, McCulloch DK, Ramsey SD, Grothaus LC. Effect of improved glycemic control on health care costs and utilization. JAMA. 2001;285(2):182-189.

34. Newton KM, Wagner EH, Ramsey SD, et al. The use of automated data to identify complications and comorbidities of diabetes: a validation study. J Clin Epidemiol. 1999;52(3):199-207.

35. Bonomi AE, Anderson ML, Rivara FP, Thompson RS. Health care utilization and costs associated with physical and nonphysical-only intimate partner violence. Health Serv Res. 2009;44(3):1052-1067.

36. Fishman PA, Goodman MJ, Hornbrook MC, Meenan RT, Bachman DJ, O'Keeffe Rosetti MC. Risk adjustment using automated ambulatory pharmacy data: the RxRisk model. Med Care. 2003;41(1):84-99.

37. Safran DG, Kosinski M, Tarlov AR, et al. The Primary Care Assessment Survey: tests of data quality and measurement performance. Med Care. 1998;36(5):728-739.

38. Bice TW, Boxerman SB. A quantitative measure of continuity of care. Med Care. 1977;15(4):347-349.

39. Smedby O, Eklund G, Eriksson EA, Smedby B. Measures of continuity of care. A register-based correlation study. Med Care. 1986; 24(6):511-518.

40. Vedsted $P$, Christensen MB. Frequent attenders in general practice care: a literature review with special reference to methodological considerations. Public Health. 2005;119(2):118-137.

41. Naessens JM, Baird MA, Van Houten HK, Vanness DJ, Campbell CR. Predicting persistently high primary care use. Ann Fam Med. 2005;3(4):324-330.

42. Diggle $\mathrm{P}$, Heagerty $\mathrm{P}$, Liang K, Zeger S. Analysis of Longitudinal Data. 2nd ed. Norfolk, UK: Oxford University Press; 2002.

43. Rodriguez HP, von Glahn T, Chang $H$, Rogers WH, Safran DG. Patient samples for measuring primary care physician performance: who should be included? Med Care. 2007;45(10):989-996.

44. Schall MW, Duffy T, Krishnamurthy A, et al. Improving patient access to the Veterans Health Administration's primary care and specialty clinics. Jt Comm J Qual Saf. 2004;30(8):415-423.

45. Murray M. Reducing waits and delays in the referral process. Fam Pract Manag. 2002;9(3):39-42

46. Kim Y, Chen AH, Keith E, Yee HF Jr, Kushel MB. Not perfect, but better: primary care providers' experiences with electronic referrals in a safety net health system. J Gen Intern Med. 2009;24(5):614-619.

47. Kilo CM. Transforming care: medical practice design and information technology. Health Aff (Millwood). 2005;24(5):1296-1301.

48. Ng M, Nathoo N, Rudnisky CJ, Tennant MT. Improving access to eye care: teleophthalmology in Alberta, Canada. J Diabetes Sci Technol. 2009;3(2):289-296

49. Reid RJ, Fishman PA, Yu O, et al. Patient-centered medical home demonstration: a prospective, quasi-experimental, before and after evaluation. Am J Manag Care. 2009;15(9):e71-e87.

50. O'Malley AS, Reschovsky JD. Referral and consultation communication between primary care and specialist physicians: finding common ground. Arch Intern Med. 2011;171(1):56-65.

51. Decker SL, Schappert SM, Sisk JE. Use of medical care for chronic conditions. Health Aff (Millwood). 2009;28(1):26-35. 